\title{
Transmisión de los valores médicos en la Universidad
}

Marcos Gómez Sancho

Universidad de Las Palmas de Gran Canaria

Debemos recordar que la posibilidad de estudio del ser humano, como fenómeno natural, ha sido una conquista fundamental en el desarrollo científico, que es a partir del Renacimiento cuando el hombre se incorpora a sí mismo como parte de la naturaleza y no como un ente totalmente separado de ella. Es gracias a dicho avance que ahora estamos en condiciones de exigir o pretender un replanteamiento de la Medicina. No se trata de un rechazo de la medicina científiconaturalista, sino de una superación de la misma.

De cualquier manera, frente al modelo "heroico" de medicina -que rechaza los límites del hombresurge hoy un modelo humanístico o paliativo que intenta comprender la vulnerabilidad, la declinación y el final de la vida humana al encuentro de una ética del cuidado.

Esta conquista metodológica cartesiana se ha mostrado de un considerable alcance que no se puede menospreciar. Pero la operatividad de las ciencias biomédicas se ha adquirido a costos bien grandes. Ellas han convertido al ser humano en un objeto. Ellas lo han objetivado. Lo han abstraído de su historia, de su subjetividad, de su existencia.

\section{EL HOMBRE ANTE LA MUERTE}

Durante más de mil años, las personas morían de una manera más o menos similar, sin grandes cambios. Era la muerte familiar. El enfermo moría en su casa, haciendo del hecho de morir, el acto cumbre de su existencia. De esta manera, era más fácil vivir la propia vida hasta el último momento, con la mayor dignidad y sentido, rodeado de los seres queridos.

La negación de la muerte, tan característica de nuestro mundo actual, ha conducido a cambios profundos y que han tenido una repercusión directa en la atención a los enfermos incurables.
En solamente una generación se ha producido un cambio espectacular en la forma de morir. Hoy en la mayoría de los países predomina la muerte en el hospital, donde es mucho más difícil "vivir la propia muerte" como un hecho consciente y digno. Otros riesgos se añaden a estas dificultades y que hacen referencia a la medicalización de la muerte. Asuntos como la eutanasia o el encarnizamiento terapéutico son algunos de los aspectos éticos que cada vez adquieren mayor relevancia en el proceso de morir, sobre todo cuando esto sucede en el hospital.

A lo largo de la historia, siempre hubo una enfermedad que para la gente tenía connotaciones mágicas, demoníacas o sagradas. Constituyen una larga secuencia desde la epilepsia, la verdadera enfermedad sagrada en tiempo de Hipócrates, quien intentó demostrar que el concepto era falso y atribuible sólo a la superstición. Después, en la antigüedad era la lepra y curarla era uno de los milagros más frecuentes en la vida de Cristo. En la Edad Media, era la sífilis y actualmente es el cáncer la enfermedad tabú. Carece del halo romántico que a principios de siglo tuvo la tuberculosis, incurable casi siempre, y comparte con la lepra y con la sífilis que no debe ser pronunciado su nombre.

Correspondiendo a las supersticiones y terrores más elementales y primitivos de la raza humana, se trata de evitar nombrar dichas enfermedades, o se pronuncia su nombre en voz baja. Los médicos utilizan eufemismos para invocarlo, la mayoría de las veces de forma incomprensible para el lego con el fin de disimular. Raramente se utiliza la palabra cáncer. Se habla, como mucho de tumor, neo, neoplasia, degeneración maligna, etc. Y en los medios de comunicación, a lo más que se llega cuando algún personaje muere de esta enfermedad es que "falleció después de una larga y penosa enfermedad". Cáncer 
equivale a mutilación y muerte y aunque es cierto que existen otros padecimientos igualmente mortales, el cáncer está considerado ahora como la enfermedad incurable por excelencia.

Lepra, peste, sífilis etc. al hacerse curables, han perdido su carácter tremendo y sagrado y estas características las ha heredado el cáncer y más modernamente, el sida.

El comportamiento del hombre ante la muerte a lo largo de la historia ha estado siempre lleno de ambigüedad, entre la inevitabilidad de la muerte y su rechazo. La conciencia de la muerte es una característica fundamental del hombre.

\section{LA MUERTE FAMILIAR}

Antaño, el hombre moría en su casa, rodeado de su familia (incluidos los niños), amigos y vecinos. Los niños tenían así contacto temprano y repetido con la muerte: primero sus abuelos, después sus padres etc. Cuando se hacía mayor y le tocaba morir a él, desde luego no le pillaba tan de sorpresa y desprovisto de recursos como sucede hoy. Hoy a los niños precisamente se les aleja de la casa cuando alguien va a morir.

Eran los momentos de los grandes amores, de los perdones y de las despedidas. Los repartos de haciendas, los últimos consejos a los hijos.

Cuando la enfermedad entraba en un momento crítico el párroco acudía a casa del feligrés llevando el viático o Eucaristía en forma procesional. La Iglesia atribuía a este sacramento numerosas virtudes: limpieza del pecado, liberación del ímpetu de las tentaciones, preparación del alma y gloria eterna. El acontecimiento, rodeado de vistosidad, llamaba a la participación popular.

Antes, generalmente, la muerte era vivida como acontecimiento público. Morir era una "ceremonia ritual" en la que el agonizante se convertía en protagonista. La muerte, aun siendo natural, se convertía en el último acto social. La "buena muerte" consistía en que, si el agonizante no advertía la llegada de los últimos momentos, esperaba que los demás se lo advirtieran para poder preparar todos sus asuntos tanto personales, como sociales y religiosos. Por el contrario, la "muerte maldita" (que se presentaba bajo una figura aterradora) era la muerte súbita (accidente, envenenamiento). Esta muerte estaba marcada por el sello de la maldición, como si unas misteriosas fuerzas demoníacas hubiesen dado origen al drama; a estas mismas fuerzas demoníacas se atribuía en la edad Media el origen de la epilepsia y la locura.
Hoy, por el contrario, las condiciones médicas en que acaece la muerte han hecho de ella algo clandestino. Ya la terapia actual en los grandes hospitales está cargada de anonimato. Anonimato que llega a su culmen en el momento de la muerte.

El caso es que hoy se oculta la muerte y se oculta todo lo que nos recuerde a ella (enfermedad, vejez, decrepitud etc.). Nada que tenga que ver con la muerte es aceptado en el mundo de los vivos.

Esto se ha traducido en un cambio radical en las costumbres y ritos funerarios y del duelo.

\section{NEGACIÓN DE LA MUERTE. MUERTE ESCAMOTEADA}

La sociedad de hoy está basada en el binomio producción-consumo y la muerte viene a anunciar el final del consumo. Por este motivo se promueve el consumo y se rechaza la muerte. La muerte aprobada socialmente ocurre cuando el hombre se ha vuelto inútil no sólo como productor, sino también como consumidor.

De esta manera ha ido cambiando la forma de morir y aquella muerte familiar, con sus ritos, etc. que acabamos de analizar, sería hoy impensable y el embate del modernismo ha introducido múltiples innovaciones. Ha cambiado el coche fúnebre, el cadáver ahora es velado en los tanatorios (ya no se llaman mortuorios), a las afueras de las ciudades, cuanto más lejos mejor. Allí se puede encontrar de todo: flores, bar, restaurante, etc. Muchos están convencidos de que algún día las ciencias biomédicas lograrán impedir la vejez y suprimir la muerte, a la que se considera una enfermedad. En los Estados Unidos existe una Comisión para la Abolición de la Muerte y en Francia se creó, en 1976, una Sociedad Inmortalista. El objetivo de ambas es promover las investigaciones que permitan prolongar la vida humana por tiempo indefinido.

\section{MEDICALIZACIÓN DE LA MUERTE. LA MUERTE EN EL HOSPITAL}

Así nos encontramos con una sociedad que, siendo mortal, rechaza la muerte. Este rechazo social a la muerte, no creo precisamente que le haya ayudado al hombre en el momento en que tiene que enfrentarse a ella. Contrasta, en efecto, este rechazo total por parte de la sociedad y la angustia, mayor que nunca, que el hombre, individualmente, siente ante ella.

Poco tiempo se tardó en averiguar cual era el sitio ideal para esconder al moribundo: el hospital. 
El hospital de hoy, es un sitio para diagnosticar y curar, y en él trabajan profesionales preparados y entrenados para diagnosticar y curar, y, por este motivo, es un mal sitio para llevar a los enfermos terminales que, por su definición, ya están diagnosticados y son incurables. El intento de domesticar el morir y la muerte puede convertir la agonía y la indigencia humanas en una situación cruel, desproporcionada, injusta e inútil, tanto para el paciente como para su familia. La muerte ha cambiado de cama. Ya no se muere en el domicilio rodeado por los seres queridos. Se han elegido los hospitales con su masificación y deshumanización para que la muerte pase desapercibida y se convierta en algo ajeno, aséptico, silencioso y solitario.

\section{EL MÉDICO ANTE LA MUERTE DE SU ENFERMO}

Existen algunos motivos por los que, a mi juicio, el médico no siempre presta suficiente atención a los enfermos terminales.

\section{FALTA DE FORMACIÓN}

Por una parte, porque en la Universidad no se nos ha enseñado nada en absoluto sobre lo que tenemos que hacer con un enfermo incurable. Por esta razón, en muchas ocasiones no se puede echar la culpa a los médicos, ya que carecen de recursos para hacer frente a las muchísimas demandas de atención que va a formular el paciente.

Los profesionales sanitarios son cada día más hábiles en el manejo de aparatos y en la utilización de técnicas complejas, pero a menudo se sienten desprovistos y desarmados de cara a la angustia y la soledad del moribundo e incapaces de establecer una relación de ayuda con él. No han sido preparados para ello.

Desgraciadamente, el hecho de no saber manejar la situación, puede dar lugar a una conducta defensiva por parte del médico y que contribuirá en gran medida a empeorar las cosas.

\section{SENSACIÓN DE FRACASO PROFESIONAL}

En segundo lugar, porque en la Universidad, como acabamos de ver, se nos ha enseñado a salvar vidas. Así, aunque sea inconscientemente, la muerte de nuestro enfermo la vamos a interpretar como un fracaso profesional, por ilógico que sea, ya que la muerte es inevitable. La muerte siempre estuvo excluida del saber médico (salvo en medicina legal).

\section{ANGUSTIA ANTE LA PROPIA MUERTE}

Parte importante de este problema, además de lo ya mencionado, es que la confrontación ante la muerte del otro nos obliga a afrontar la realidad, tantas veces negada, de la propia muerte.

Los profesionales de la salud, antes que doctos eruditos, somos seres humanos con las mismas características fundamentales que aquel que yace esperando nuestro cuidado: somos de la misma y perecedera materia. Es en escenarios como este, donde afloran nuestros prejuicios y creencias (las propias y las inculcadas a lo largo de nuestra formación profesional), al igual que nuestras ansiedades y temores de muerte y nuestra propia historia personal. Cuanto más semejanza perciba entre el enfermo y sí mismo, más relevante será el problema (por ejemplo, cuando el médico se encuentra ante un miembro de su familia, un colega, una persona de su edad, etc.).

Los médicos somos casi los únicos, en nuestra comunidad, a quienes se nos atribuye la inmortalidad, la omnipotencia y la falta de sentimientos, aunque la verdad sea muy distinta. No hay cosa más curiosa y digna de meditación que el inocente asombro de la gente porque el médico está enfermo o porque el médico está emocionado.

Todo profesional de la salud debería tener interés en analizar y comprender los diferentes componentes de su malestar. Una toma de conciencia de las razones ocultas que le empuja a huir ante tales situaciones, permite a veces rectificar su actitud y estar más cómodo en semejantes circunstancias.

Se ha confundido la misión tradicional del médico, esto es, aliviar el sufrimiento humano y que en líneas generales se puede expresar de la siguiente manera:

Si puedes curar, cura.

Si no puedes curar, alivia.

Y si no puedes aliviar, consuela.

Aliviar y consolar es con frecuencia lo único que podemos hacer por ayudar al enfermo, pero que no es poco. El hecho de que al enfermo no se le considere muerto antes de morir, que no se considere abandonado por su médico, que le visita, le escucha, le acompaña, le tranquiliza y conforta, le da la mano y es capaz de transmitirle esperanza y confianza, es de una importancia tremenda para el paciente, aparte de una de las misiones más grandiosas de la profesión médica, profesión que posee la humilde grandeza de tener al Hombre como objeto. El médico tiene que estar ahí —cueste lo que cueste, porque la muerte es índice de su fracaso, tal 
y como hoy se entiende-, para ayudarle a morir. Ser médico es, en primer lugar, ser nada más que médico, y al mismo tiempo, ser médico hasta el final. Ningún médico está autorizado a abandonar a su enfermo por el mero hecho de padecer una enfermedad incurable y grave.

El médico debe aprender, por fin, que la muerte es algo natural. Cuando el médico rechaza la muerte, termina por abandonar al enfermo; cuando la niega y se niega a dejar morir a su enfermo, caerá en el encarnizamiento o furor terapéutico (intento curativo persistente). Solamente cuando es capaz de aceptarla como algo natural y, antes o después, inevitable, se dedicará a cuidar a su enfermo hasta el final y sin sensación de fracaso. La muerte es el precio que paga todo ser pluricelular desde el mismo momento de su nacimiento.

\section{HUMANISMO Y RELACIÓN MÉDICO-ENFERMO}

El humanismo es un arte de palabras, sentimientos y actitudes. El médico lo expresa con empatía, poniéndose genuinamente y con afecto en la realidad ajena, lo que a su vez evoca en el paciente confianza, seguridad y esperanza.

A pesar de ello, cada vez es mayor el número de personas que se quejan de la ausencia de humanidad en el médico actual. Los enfermos añoran la imagen del médico benevolente y comprensivo de antaño a pesar de que con frecuencia no podía hacer otra cosa que ser un testigo del curso de una enfermedad para la que no disponía de ningún remedio.

Los avances en la tecnología diagnóstica han disminuido considerablemente la necesidad de entrevistas clínicas minuciosas. Como resultado, la relación médico-enfermo se establece prioritariamente a través de procedimientos y aparatos, a los que comprensiblemente se les atribuyen los beneficios "tangibles" y "reales" de la intervención médica.

A estos factores distanciantes entre el médico y el enfermo hay que añadir la influencia deshumanizante de la cultura del trabajo en el sistema sanitario saturado y agobiante de nuestras ciudades. Muchos médicos de hoy son funcionarios renuentes, mal retribuidos y atrapados en un mundo tecnocrático que odian. Se sienten acosados por administradores impacientes por controlar y por burócratas ansiosos por regular, y están siempre faltos de energía para sentarse a la cabecera del doliente e impartirle esperanza.

Otro hecho incuestionable es que en la mayoría de las instituciones públicas actuales los enfermos son considerados números y no individuos, vidas estadísticas sin identidad. La estructura sanitaria está organizada principalmente para satisfacer la mecánica interna de la institución y la conveniencia del personal, y no para el beneficio del enfermo.

Uno de los factores determinantes de la carencia de humanismo de la medicina actual radica en la perversión de los esquemas económicos. A los médicos se les prima por atender al mayor número de enfermos en el menor tiempo posible, por utilizar procedimientos técnicos avanzados, por ordenar pruebas de laboratorio y por intervenir quirúrgicamente.

Nadie duda de que hay algo fundamental que falla en la medicina de hoy. Fría, cara e incluso cruel, la asistencia sanitaria plantea un desafío a los médicos a la hora de armonizar la efectividad de la ciencia con el humanismo de la empatía. El camino será arduo pero el reto es necesario .

Teniendo en cuenta todos estos argumentos, sería equivocado identificar la competencia profesional de un médico con su competencia técnica. La capacidad científico técnica es una condición necesaria, pero no suficiente, para la competencia. Es necesario sumar la calidad humana para alcanzar un resultado suficiente. El concepto de "calidad humana" englobaría una serie de factores y realidades que no se dejan atrapar por la ciencia biológica: relación de ayuda y confianza (relación clínica), consideración de la dignidad y preferencias del paciente (aspectos éticos), contexto familiar y social (aspectos comunitarios) .

Todo lo dicho hasta aquí, respecto a la deshumanización y fragmentación de la medicina moderna altamente tecnificada, el deterioro de la relación médico-enfermo, los "hospitales inhóspitos", la insatisfacción y descontento de los usuarios etc. adquiere, como es lógico, una importancia decisiva en los pacientes en situación terminal, que ya no van a necesitar casi nunca de las ventajas de la alta tecnología y sin embargo van a sufrir con frecuencia todos sus inconvenientes.

\section{BIBLIOGRAFÍA}

1. Gómez Sancho M. El Hombre y el Médico ante la muerte. Madrid: Arán, 2006.

2. Rojas Marcos L. Medicina y empatía. Jano 1995; 1125: 625

3. Altisent R. Humanidades para médicos. Jano 1997; 1202: 9 . 
Documento propuesto en la Subcomisión de Ética del Comité Permanente de Médicos Europeos. Bruselas. Elaborado por la Comisión "Los Valores de la Medicina del siglo XXI" de la Organización Médica Colegial de España. Coordinador: Marcos Gómez Sancho, Rogelio Altisent, Jacinto Batiz, Luis Ciprés Casasnovas, Pablo Fernández Gutiérrez, José Antonio Herranz Martínez, Manuel Pérez Martí, Joan Viñas

La reflexión sobre los fines y las prioridades de la medicina lleva de la mano al análisis crítico de la formación universitaria. No hay duda de que los médicos europeos reciben una buena formación de pregrado y postgrado, lo cual viene avalado por el reconocimiento de los diferentes programas de formación de médicos y especialistas.

Sin embargo, también hay acuerdo en la necesidad de abordar reformas encaminadas a lograr un perfil de formación médica más centrada en la dimensión humana del paciente y en sus necesidades como persona. No se trataría tanto de incorporar nuevas áreas de conocimiento como de introducir una filosofía de la medicina más integradora y humanista que impregne con eficacia todo el curriculum.

Existe la percepción de que el estudiante de medicina necesita terminar la licenciatura con un mayor grado de madurez para comprender el significado que la salud y la enfermedad tienen para el individuo; el alcance de las preferencias y valores del paciente y los límites de la ciencia tanto en la investigación como en las aplicaciones clínicas. Todo esto requiere estrategias docentes adecuadas y una intencionalidad muy decidida en el profesorado.

El imparable y progresivo avance científico-técnico experimentado por la medicina en las últimas décadas se ha traducido en un proceso de superespecialización que ha tenido como efecto negativo la fragmentación conceptual del paciente y el riesgo de despersonalización en la atención médica.

Las necesidades de los seres humanos en relación con su salud van más allá del simple modelo de curación de la enfermedad donde el médico hace el diagnóstico y prescribe una terapia. La comprensión del paciente en su modo de reaccionar ante la enfermedad y el sufrimiento, exige del médico una serie de actitudes enfocadas a la ayuda y al servicio de las personas.

El progresivo incremento de las enfermedades crónicas constituye actualmente un paradigma que ya no se puede considerar como cuestión marginal en la enseñanza de las Facultades de Medicina. No es lo mismo aprender a tratar a un enfermo agudo que a uno crónico, del mismo modo que hay diferentes prioridades asistenciales entre el paciente que se encuentra en cuidados intensivos y el que está en situación de enfermedad terminal. La demanda social de medicina paliativa es un buen ejemplo para entender la urgencia de reformas curriculares más adaptadas a las necesidades de la sociedad.

Otro fenómeno a tener en cuenta es el creciente protagonismo de la dimensión preventiva de la medicina y el impacto del estilo de vida en la promoción de la salud, lo cual sitúa al médico ante fun- ciones de consejero y educador, e incluso de comunicador social. Las medidas de salud pública y su impacto deberían estudiarse de un modo más relacionado con la asistencia individual.

La sociedad espera de los médicos un sincero y efectivo respeto hacia los derechos y valores del paciente, lo cual requiere desarrollar actitudes y habilidades de comunicación en la relación clínica para sintonizar, informar adecuadamente y obtener su consentimiento, promoviendo su participación en la toma de decisiones. De hecho, la conveniencia de incorporar al curriculum médico contenidos académicos de ética ya está fuera de discusión.

El entrenamiento profesional para relacionarse con la familia como microcosmos comunitario juega un papel decisivo en la promoción de la salud de sus miembros y en el proceso de cuidado del enfermo. Esto resulta especialmente importante en la atención domiciliaria, cada vez más necesaria y demandada por la sociedad.

Desde hace ya años el trabajo interdisciplinar y de equipo en el campo de la salud se ha convertido en una necesidad para la que se requiere actitudes y capacitación que deberían enseñarse en la licenciatura. La coordinación del mundo sanitario con los servicios sociales es un desafío que debe tener una presencia relevante en las aulas universitarias al tratar el fenómeno socio-sanitario de la dependencia.

Por otro lado, la necesidad de modelos de organización sanitaria que garanticen la equidad exige que en la formación médica estén presentes conceptos relacionados con la gestión de recursos: eficiencia, conciencia del coste, sostenibilidad del sistema sanitario, etc.

Se hace necesario un mayor esfuerzo e ingenio docente para introducir las ciencias sociales y las humanidades de forma transversal, impregnando los contenidos biomédicos de un modo que resulte atractivo para los estudiantes.

Estos contenidos relacionados con la dimensión humana y social de la medicina deberían estar presentes ya desde el primer año de la licenciatura, motivando al estudiante con el análisis y la reflexión de casos o situaciones donde pueda desarrollar actitudes que están en el corazón de la profesión médica. Del mismo modo convendría potenciar las cualidades humanas del buen médico (compasión, cortesía, calidez, etc.).

La responsabilidad social de la institución universitaria exige una gran capacidad de adaptación a las necesidades cambiantes de la comunidad. La innovación curricular que se propone debe ser compatible con la solidez científica propia de una Facultad de Medicina. Y este desafío ha de estar presente en las agendas de los claustros académicos. 